\title{
Impact of COVID-19 lockdown on progressive multiple sclerosis patients
}

\author{
Marco Vercellino $^{1}$ (D) Chiara Bosa $^{1} \cdot$ Anastasia Alteno $^{1} \cdot$ Valentina Schillaci $^{1} \cdot$ Manuel Petracca $^{1} \cdot$ Stella Marasciulo $^{1}$. \\ Paola Cavalla ${ }^{1}$
}

Received: 31 October 2021 / Accepted: 19 January 2022 / Published online: 24 January 2022

(c) Fondazione Società Italiana di Neurologia 2022

\begin{abstract}
Introduction The COVID-19 pandemic caused major changes in the lifestyle and in the access to health services worldwide. Progressive multiple sclerosis (pMS) patients are a vulnerable population at high risk of disability worsening.

Objective and Methods The objective of this study was to assess the health outcomes of COVID-19 lockdown in a cohort of 225 pMS patients.

Results Worsening of neurological disability (19.7\%) and fatigue (32.4\%), depression (30.4\%), and weight increase (28.3\%) were observed in pMS patients during lockdown, along with discontinuation of regular physical exercise (47.1\%) and of physical therapy $(59.3 \%)$.

Conclusion These results highlight the adverse impact, on pMS patients, of the public health measures implemented for the containment of the pandemic.
\end{abstract}

Keywords COVID-19 $\cdot$ Progressive multiple sclerosis $\cdot$ Lockdown $\cdot$ Fatigue $\cdot$ Exercise $\cdot$ Disability

\section{Introduction}

The COVID-19 pandemic caused major changes in the lifestyle and in the access to health services for many people worldwide. Healthcare resources have been rapidly redirected to emergency and intensive care settings, while access to outpatient services has been limited both to avoid the spread of the pandemic and to free resources for care of COVID-19 patients [1].

Progressive multiple sclerosis (pMS) patients are a vulnerable population at high risk of disability worsening, and potentially facing more adverse consequences as a result of the disruption of their normal lifestyle and of the difficulties in the access to health services. pMS patients are also at higher risk for a severe course of COVID-19 infection;

Marco Vercellino

marco.vercellino@unito.it

1 Multiple Sclerosis Center, Neurologia I U, Department of Neuroscience and Mental Health, AOU Città della Salute e della Scienza di Torino, Via Cherasco 15, 10126 Torino, Italy during the pandemic, they were therefore generally advised to carefully enforce social distancing and to carry out strict precautionary measures to avoid contagion [2].

Northern Italy was one of the most heavily hit regions of Europe in the first wave of the COVID-19 pandemic; consequently, a nationwide hard lockdown was imposed from the beginning of March 2020, and eased by the end of May 2020. As in many other nations throughout the world during the pandemic, lockdown regulations severely restricted normal daily activities save for those not strictly essential. People were supposed to stay home and go outside only if absolutely necessary. During lockdown, and also in the following months, access to outpatient services was difficult and physical therapy services were closed, both because of safety reasons (containment of viral spread) and because hospitals were burdened with a high number of COVID-19 patients, while rehabilitation clinics had been converted to post-hospital care for discharged COVID-19 patients [1,2].

Objective of this study was to assess and describe the health outcomes of COVID-19 lockdown in a cohort of pMS patients. 


\section{Methods}

All pMS patients followed in the MS Center - AOU Città della Salute e della Scienza di Torino - Italy, were included. The only inclusion criteria was pMS, without any exclusion criteria except patients living outside of Italy at the moment of lockdown and therefore not experiencing lockdown. pMS was defined by documented disability progression in the 2 previous years and presence of at least moderate disability (EDSS $\geq 3.0$ ), with or without a previous history of relapsing MS.

During the lockdown period (from the beginning of March 2020 to the end of May 2020), patients were not seen at the MS Center, but only assessed by telehealth, except for emergencies or scheduled infusional therapies. Regular visits in the MS Center were resumed in June 2020. Clinical, MRI, and anamnestic data were obtained from the records of the MS Center and from prospective follow-up. Self-reported regular physical exercise and engagement in physical therapy, self-reported worsening of fatigue and perceived disability, and self-reported worsening of depressive symptoms were collected by patient interview by the treating neurologist, during the first visit at the MS center after lockdown (within 3 months after the end of lockdown). EDSS assessment was performed at the same time and confirmed during a follow-up visit after 6 months. The reference period before lockdown was the 12 months preceding lockdown. The reference EDSS was the one assessed during the last visit before lockdown (within 6 months before lockdown). Regular physical exercise was defined as a subset of physical activity that is planned, structured, and repetitive and has as an objective the improvement or maintenance of physical fitness [3] (i.e., walking $>30 \mathrm{~min} /$ day, exercise bike, free body workout...). Regular engagement in physical therapy was defined as a regular engagement in therapy for the preservation, enhancement, or restoration of movement and physical function impaired by disease, under supervision of a physical therapist, at least once a week. Depressive symptoms assessed during the interview by the treating neurologist included depressed mood (persistent feelings of sadness and hopelessness) and loss of interest or pleasure in activities [4]. We also collected data on all relapses in 2020, as well as MRI data on all patients with a MRI performed in 2020: the purpose of this was to describe the studied population in terms of disease activity.

Classic descriptive statistics indicators (mean with standard deviation and percentiles for continuous variables and percentages for categorical ones) with relative $95 \%$ confidence intervals were estimated in order to describe the studied population. The Mann-Whitney U test was used to compare differences between groups. The association between subjective self-reported assessment (self-reported worsening of perceived disability) and objective assessment (EDSS) was evaluated using a chi-square test.

\section{Results}

This cohort included 225 pMS patients: $25 \%$ primary progressive, $75 \%$ secondary progressive; male to female ratio 0.76 ; mean age $57.6 \pm 10.3$ years, mean duration of disease $17.7 \pm 10.6$ years, median EDSS 6.0 (range 3.0-9.0); firstline therapy $18.2 \%$, second-line therapy $35.1 \%$ (mostly anti CD20, 28.4\%), no treatment $45.3 \%$ (Supplementary Table 1).

A perceived worsening of disability and fatigue during/after lockdown (compared to the 12 months prior to lockdown) was reported by $32.4 \%$ of patients. A total of $30.4 \%$ of patients reported worsening of depressive symptoms during lockdown (compared to the 12 months prior to lockdown). Increase of the EDSS score $\geq 0.5$, confirmed at 6 months, was observed in $19.7 \%$ of patients at the followup visits after the period March 2020-May 2020 (mean EDSS increase $0.66 \pm 0.26$ ), compared to $9.0 \%$ of patients in the 12 months before March 2020. A close association was observed between self-reported worsening of disability and objective EDSS worsening $(p<0.0002)$. No significant differences in age or gender were observed in patients with or without self-reported worsening of disability or objective EDSS worsening. MS relapses were infrequent, as was MRI evidence of disease activity (defined by new/ enlarging T2 lesions or T1 Gd+ lesions in 2020, compared to a reference scan in the 24 months before 2020): 4 out of 225 patients experienced a MS relapse in 2020, and 13 patients showed MRI disease activity out of 132 patients who underwent MRI in 2020 (13 with new/enlarging T2 lesions, none with $\mathrm{T} 1 \mathrm{Gd}+$ lesions).

Before lockdown, many pMS patients reported regular physical exercise (42.8\%). An active lifestyle and regular physical exercise were routinely promoted as a part of disease management by the MS Center. Many patients regularly practiced physical therapy $(46.2 \%)$, followed by a physical therapist at home or in an outpatient setting. Regular physical exercise was predominantly reported by pMS patients with relatively lower disability (mean EDSS 5.23 vs 6.40 , $p<0.001$ ), while patients with higher disability were less likely to practice physical exercise and more likely to do physical therapy (mean EDSS 6.18 vs 5.28, $p<0.001$ ); $19.4 \%$ reported both. Most patients $(59.3 \%)$ had to completely stop physical therapy during lockdown, and many patients $(47.1 \%)$ completely ceased physical exercise. All patients found their movements outside home severely restricted: the lockdown regulations stated that all unnecessary outdoor activities were banned. A weight increase during lockdown was observed in $28.3 \%$ of patients $($ mean $+4.5 \mathrm{~kg} \pm 2.7)$, while $9.1 \%$ showed a weight decrease.

In the 6 months after lockdown, only $42.6 \%$ of patients resumed physical exercise (among those who already 
practiced regular exercise before lockdown), and only $63.2 \%$ could resume physical therapy, as outpatient services mostly remained closed.

COVID-19 infection was reported in 8/225 patients, with need of hospitalization only for 3 patients. No patient died of COVID-19.

\section{Discussion}

During the COVID-19 pandemic, several public health measures, colloquially known as lockdown, were implemented in many countries worldwide; these included stayat-home orders, social distancing, self-quarantine, and curfews. There are concerns that the lockdown measures have encouraged a shift toward a more physically inactive lifestyle [5]. Studies have reported a decrease in physical activity and an increase in sedentary behaviors during lockdown [5]. Adverse mental health consequences of the lockdown have also been reported in several studies [6].

pMS patients are a vulnerable population at high risk of disability worsening. Regular physical exercise can improve endurance and muscle mass/strength, helping to compensate for the effects of neurological disability [7, 8]. Fatigue is a common symptom in pMS; several studies suggest that regular exercise in MS patients can improve walking and balance, improve fatigue, and improve quality of life [7, 8]. Physical therapy interventions can improve spasticity, joint pain/stiffness, and mobility [9]. In our cohort, a decrease in physical activity and increase in sedentary behaviors was very common in pMS patients during lockdown, and also in the following months, possibly contributing to increased perceived and observed disability.

pMS patients are also a population with high prevalence of depressive disorders, and potentially at higher risk to experience adverse mental health consequences during lockdown. Previous studies have explored the psycho-social consequences of COVID-19 lockdown on the broad population of MS patients, highlighting the increase of stress and anxiety [10-13].

Several studies have shown a negative effect of lockdown on eating habits, with an increase in food consumption and reduction in physical activity with a consequent increase in weight $[5,14,15]$. Overweight in pMS patients can adversely impact neurological disability and fatigue. This study has several limitations, being a collection of data on outcomes on a pMS cohort after a dramatic and unforeseen event such as the COVID-19 lockdown, and not a pre-planned prospective study assessing patients over a predetermined time period. As such, it was mainly based on self-reported outcomes and did not employ structured scales except for the EDSS.
In conclusion, worsening of neurological disability, worsening of fatigue and depression, and weight gain were observed in a sizable proportion of pMS patients during COVID-19 lockdown, along with a shift to a more inactive lifestyle and difficulty to continue regular physical therapy. These results highlight the adverse impact, on pMS patients, of the public health measures implemented for the containment of the pandemic.

Supplementary Information The online version contains supplementary material available at https://doi.org/10.1007/s10072-022-05909-z.

Author contribution All Authors contributed to the study conception and design. Material preparation, data collection, and analysis were performed by Paola Cavalla, Chiara Bosa, Anastasia Alteno, Valentina Schillaci, Manuel Petracca, Stella Marasciulo, and Marco Vercellino. The first draft of the manuscript was written by Marco Vercellino and all authors commented on previous versions of the manuscript. All authors read and approved the final manuscript.

Data availability The datasets analyzed during the current study are available from the corresponding author on reasonable request.

\section{Declarations}

Ethical approval This observational study was approved by the local Ethical Committee (Comitato Etico Interaziendale A.O.U. Città della Salute e della Scienza di Torino, A.O. Ordine Mauriziano, A.S.L. Città di Torino).

Conflict of interest The authors declare no competing interests.

Informed consent statement All involved persons gave their informed consent for inclusion and use of their personal and clinical data in the study.

\section{References}

1. The impact of the COVID-19 pandemic on noncommunicable disease resources and services: results of a rapid assessment. World Health Organization (WHO), September 3rd, 2020. https://www. who.int/publications/i/item/9789240010291. Accessed 30 Oct 2021

2. Manacorda T, Bandiera P, Terzuoli F et al (2021) Impact of the COVID-19 pandemic on persons with multiple sclerosis: early findings from a survey on disruptions in care and self-reported outcomes. J Health Serv Res Policy 26(3):189-197. https://doi. org/10.1177/1355819620975069

3. Caspersen CJ, Powell KE, Christenson GM (1985) Physical activity, exercise, and physical fitness: definitions and distinctions for health-related research. Public Health Rep 100(2):126-31

4. American Psychiatric Association (2013) Diagnostic and statistical manual of mental disorders (5th ed.). https://doi.org/10.1176/ appi.books. 9780890425596

5. Ammar A, Brach M, Trabelsi K et al (2020) Effects of COVID19 home confinement on eating behaviour and physical activity: results of the ECLB-COVID19 International Online Survey. Nutrients 12(6):1583. https://doi.org/10.3390/nu12061583 
6. Brooks SK, Webster RK, Smith LE et al (2020) The psychological impact of quarantine and how to reduce it: rapid review of the evidence. Lancet 395(10227):912-920. https://doi.org/10.1016/ S0140-6736(20)30460-8

7. Motl RW, Sandroff BM, Kwakkel G et al (2017) Exercise in patients with multiple sclerosis. Lancet Neurol 16(10):848-856. https://doi.org/10.1016/S1474-4422(17)30281-8

8. Heine M, van de Port I, Rietberg MB et al (2015) Exercise therapy for fatigue in multiple sclerosis. Cochrane Database Syst Rev (9):CD009956. https://doi.org/10.1002/14651858.CD009956. pub2

9. Haselkorn JK, Hughes C, Rae-Grant A et al (2015) Summary of comprehensive systematic review: rehabilitation in multiple sclerosis: report of the Guideline Development, Dissemination, and implementation subcommittee of the American Academy of Neurology. Neurology 85(21):1896-1903. https://doi.org/10. 1212/WNL.0000000000002146

10. Motolese F, Rossi M, Albergo G et al (2020) The psychological impact of COVID-19 pandemic on people with multiple sclerosis. Front Neurol 30(11):580507. https://doi.org/10.3389/fneur.2020. 580507

11. Morris-Bankole H, Ho AK (2021) The COVID-19 Pandemic experience in multiple sclerosis: the good, the bad and the neutral. Neurol Ther 10(1):279-291. https://doi.org/10.1007/ s40120-021-00241-8

12. Bonavita S, Sparaco M, Russo A, Borriello G, Lavorgna L (2021) Perceived stress and social support in a large population of people with multiple sclerosis recruited online through the COVID-19 pandemic. Eur J Neurol 28(10):3396-3402. https://doi.org/10. 1111/ene. 14697

13. Costabile T, Carotenuto A, Lavorgna L, Borriello G, Moiola L, Inglese M, Petruzzo M, Trojsi F, Ianniello A, Nozzolillo A, Cellerino M, Boffa G, Rosa L, Servillo G, Moccia M, Bonavita S, Filippi M, Lanzillo R, Brescia Morra V, Petracca M (2021) COVID-19 pandemic and mental distress in multiple sclerosis: implications for clinical management. Eur J Neurol 28(10):33753383. https://doi.org/10.1111/ene. 14580

14. Thompson AJ, Baranzini SE, Geurts J, Hemmer B, Ciccarelli O (2018) Multiple sclerosis. Lancet 391(10130):1622-1636. https:// doi.org/10.1016/S0140-6736(18)30481-1

15. Esposito S, Bonavita S, Sparaco M, Gallo A, Tedeschi G (2018) The role of diet in multiple sclerosis: a review. Nutr Neurosci 21(6):377-390. https://doi.org/10.1080/1028415X.2017.1303016

Publisher's note Springer Nature remains neutral with regard to jurisdictional claims in published maps and institutional affiliations. 